\title{
Properties of the electron cloud in a high-energy positron and electron storage ring
}

\author{
K. C. Harkay* and R. A. Rosenberg ${ }^{\dagger}$ \\ Advanced Photon Source, Argonne National Laboratory, Argonne, Illinois 60439
}

(Received 13 November 2002; published 20 March 2003)

\begin{abstract}
Low-energy, background electrons are ubiquitous in high-energy particle accelerators. Under certain conditions, interactions between this electron cloud and the high-energy beam can give rise to numerous effects that can seriously degrade the accelerator performance. These effects range from vacuum degradation to collective beam instabilities and emittance blowup. Although electron-cloud effects were first observed two decades ago in a few proton storage rings, they have in recent years been widely observed and intensely studied in positron and proton rings. Electron-cloud diagnostics developed at the Advanced Photon Source enabled for the first time detailed, direct characterization of the electroncloud properties in a positron and electron storage ring. From in situ measurements of the electron flux and energy distribution at the vacuum chamber wall, electron-cloud production mechanisms and details of the beam-cloud interaction can be inferred. A significant longitudinal variation of the electron cloud is also observed, due primarily to geometrical details of the vacuum chamber. Such experimental data can be used to provide realistic limits on key input parameters in modeling efforts, leading ultimately to greater confidence in predicting electron-cloud effects in future accelerators.
\end{abstract}

DOI: 10.1103/PhysRevSTAB.6.034402

PACS numbers: 29.27.Bd, 29.30.Aj, 41.75.Ht

\section{INTRODUCTION}

In recent years, a growing number of observations of electron-cloud effects have been reported in various positron and proton rings [1-6], in some cases after operating them in a new configuration [7]. These observations give an indication that low-energy, background electrons in high-energy particle accelerators are very common. In many cases, the electrons are not detrimental to accelerator performance; however, there are operating conditions that can lead to large amplification of the electron cloud. The cloud electrons can be produced directly by ionization of the residual gas and by irradiation of the vacuum chamber surface by synchrotron radiation, ions, or beam particles. This is denoted the primary component of the cloud. Indirectly, bombardment of the chamber surface by electrons accelerated by the beam can lead to production of secondary electrons (SEs). This is denoted the secondary component of the cloud; a comprehensive review of this secondary electron generation can be found in Ref. [8]. The distribution of the electron cloud (EC) will depend on which processes dominate in a given ring. If the cloud density becomes sufficiently large, the beamcloud interaction can degrade the particle beam. ECinduced effects range in severity from noise on beam diagnostics and vacuum degradation through electronstimulated gas desorption to transverse beam collective instabilities. Instabilities can take two forms: coupledbunch instabilities caused by a wakefieldlike interaction $[1,2]$ or single-bunch instabilities in a mechanism similar to the strong head-tail instability [9], resulting primarily in emittance blowup. A more complete discussion of

\footnotetext{
*Email address: harkay@aps.anl.gov

†Email address: rar@aps.anl.gov
}

electron-cloud effects can be found in review articles by Furman [10], Zimmermann [11], and Arduini [12].

In order to directly measure the properties of the electron cloud, a special chamber was installed at the Advanced Photon Source (APS) storage ring, equipped with compact electron energy retarding field analyzers (RFAs) [13]. Standard beam position monitors (BPMs) were also installed on the chamber for comparison. The RFA measures the electron flux at the chamber wall as a function of integrated electron energy. Analyses of the measured electron-cloud distributions have provided an enhanced understanding of the details of the cloud production and beam-cloud interaction. Prior to the development of electron-cloud diagnostics, the presence of electrons had to be inferred indirectly either by an anomalous vacuum pressure rise, by noise induced on beam electronics, or by qualitative similarities between theoretical instability predictions and beam experiments. Such indirect evidence was not always entirely convincing [4]. Dedicated electron detectors based on the APS RFA are now widely implemented $[3,4,6]$.

The experimental studies at the APS were designed to address two issues: To characterize the EC distribution for better prediction of machine conditions leading to collective instabilities and other cloud-induced effects and to identify and provide realistic limits on key ingredients in computer models of electron-cloud production. Key factors contributing to the electron cloud include chamber surface characteristics such as roughness, photoelectron and secondary electron yield coefficients, and secondary electron distribution; machine parameters such as bunch current and spacing, and the presence of an external electric or magnetic field; and vacuum chamber geometry, especially the presence of an antechamber and synchrotron radiation absorbers. 
The major results of the APS studies [13-15] can be summarized as follows. First, a dramatic amplification was observed in the electron cloud for positron beams with a $20-\mathrm{ns}$ bunch spacing ( $7 \mathrm{rf}$ wavelengths, $\lambda_{\mathrm{rf}}$ ). This gain is attributed to beam-induced multipacting (BIM), and was accompanied by an anomalous vacuum pressure rise, consistent with observations in the CERN Intersecting Storage Ring [16]. EC amplification was also observed for electron beams, but with a $30-n s\left(11 \lambda_{\text {rf }}\right)$ bunch spacing. The measured amplification of the cloud, however, is more modest with an electron beam. At the resonance condition, the electron-cloud density rises exponentially over the bunch train until a saturation limit; the saturation level and bunch number at which the limit is reached were observed to depend nonlinearly on the bunch current. In addition, what appears to be a cloudinduced horizontal coupled-bunch instability was observed for positrons with about $2 \mathrm{~mA}(7 \mathrm{nC})$ per bunch. This instability has not been observed for electron beams at identical operating conditions. Finally, the measured electron-cloud signals decrease over time as the chamber surfaces are irradiated, giving evidence of a beaminduced conditioning effect. This decrease is consistent with bench measurements showing the reduction of the secondary electron yield coefficient $(\delta)$ under electron or photon bombardment [17-19]. It is noted that both BIM and electron-cloud instabilities could be important in both positively and negatively charged beams, albeit at a higher threshold in the latter case. For the present machine configuration, electron-cloud effects do not limit the performance of the APS storage ring, now operating with electron beams.

The APS storage ring operating parameters are described first, including a brief chronology of EC studies. This is followed by details of the experimental setup. The results of the measurements are described next, which include data for positron and electron beams. Finally, the effects of surface conditioning and an estimate of the electron-cloud density in the chamber is discussed.

\section{APS STORAGE RING}

The 7-GeV APS x-ray synchrotron light source was operated for two years with a positron beam (19961998), after which it was converted to an electron beam operation. The vacuum chamber is made of $\mathrm{Al}$, which has a relatively high $\delta$ [18] due to oxidation of the surface. This presented a unique opportunity to study electroncloud effects with both positron and electron beams. Typical machine operating parameters are given in Table I.

Prior to dedicated studies, no EC effects were observed for nominal, 100-mA user operation conditions. By chance, bunch fill patterns avoided the BIM resonance conditions. With positron beams, the machine had been operated either with many, low-current bunches at $1-\lambda_{\mathrm{rf}}$
TABLE I. Typical parameters for APS electron-cloud studies.

\begin{tabular}{lcc}
\hline \hline Circumference & 1104 & $\mathrm{~m}$ \\
Beam energy & 7 & $\mathrm{GeV}$ \\
Harmonic number & 1296 & \\
rf frequency & 351.93 & $\mathrm{MHz}$ \\
Bunch charge (current) & $3.7-11 \mathrm{nC}$ & $(1-3 \mathrm{~mA})$ \\
rms bunch length & 7 & $\mathrm{~mm}$ \\
Transverse rms sizes & 350,20 & $\mu \mathrm{m}$ \\
Vacuum chamber semiaxes & $42.5,21$ & $\mathrm{~mm}$ \\
Antechamber slot height & 10 & $\mathrm{~mm}$ \\
Vacuum chamber material & $\mathrm{Al}$ & \\
\hline \hline
\end{tabular}

spacing, or few, high-current bunches at large spacing (54 $\lambda_{\text {rf }}$ ). A BIM resonance condition was found experimentally ( $7-\lambda_{\text {rf }}$ bunch spacing) and systematically studied over a period of operation. Electron-cloud buildup and saturation were also studied over long bunch trains both on and off the BIM resonance. A horizontal coupledbunch instability was observed to occur with about $2 \mathrm{~mA}$ (7 nC) per bunch at the BIM condition. Unfortunately, only a limited amount of time was available to study this instability in detail before the storage ring was converted to electron beam operation. With electron beams, beam lifetime degradation has been observed with certain bunch fill patterns due to vacuum degradation, and this is well correlated with measured EC amplification. There is no indication of an electron-cloud-induced instability for bunch currents up to $2 \mathrm{~mA}$ for electron beams; however, we are investigating whether instabilities may exist with higher bunch currents. That electron beams may also be affected by electron-cloud effects was first proposed by Galayda [20]. We have plans to continue studying both ion effects and electron-cloud-induced effects with electron beams.

\section{EXPERIMENTAL SETUP}

A special 5-m vacuum chamber, equipped with ten RFAs [13] and three BPMs, was built and installed in a field-free region in the APS storage ring [14]. The locations of the components are shown in Fig. 1. In the figure, EA is a water-cooled, copper end absorber designed to intercept high-energy photons to protect the downstream structures. The detail in Fig. 2 shows a schematic of the synchrotron radiation fan from the upstream bending magnet. The horizontal opening angle is determined by the position of the upstream photon absorber and the bending magnet radius. The EA is a significant, local source of primary electrons.

The APS electron energy analyzer is based on a planar RFA geometry and is described in detail elsewhere $[13,21]$. The RFA consists of a grounded outer grid, a second, shielded grid that can be biased, and a graphitecoated collector; a schematic is shown in Fig. 3. The detector is mounted behind a vacuum penetration slotted 


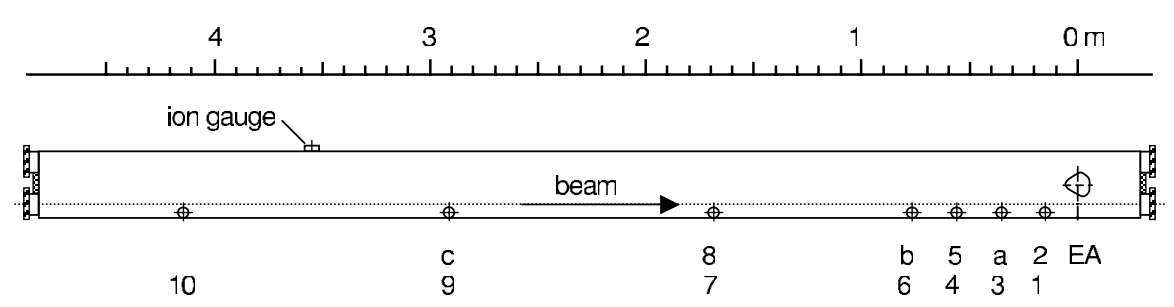

FIG. 1. Modified vacuum chamber (top view) showing locations of RFA detectors 1-10 and BPMs $a, b$, and $c$. The top numbers and letters indicate those detectors or BPMs mounted above the chamber midplane; the bottom numbers indicate those mounted below. The end absorber EA intercepts high-energy photons.

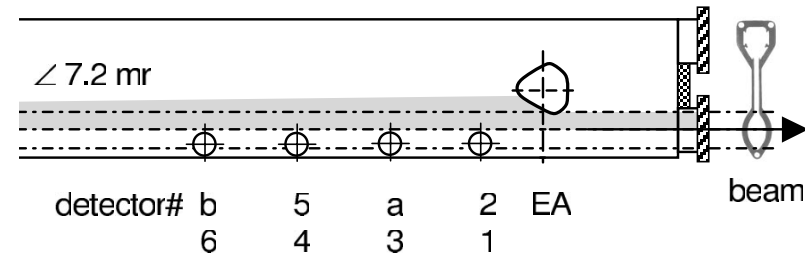

FIG. 2. Figure 1 detail showing the synchrotron radiation fan.

for $\mathrm{rf}$ shielding of the beam image current. Figure 4 shows two detectors mounted on the standard-aperture APS vacuum chamber. The antechamber channel allows most of the high-energy photons to escape without colliding with the chamber walls. A standard BPM is mounted opposite a detector at three locations for comparison. The BPM surface area and the detector aperture are both about $1 \mathrm{~cm}^{2}$. The approximate limits of the radiation fan at several detector locations are given by the rectangles drawn in Fig. 4. The vertical limit of

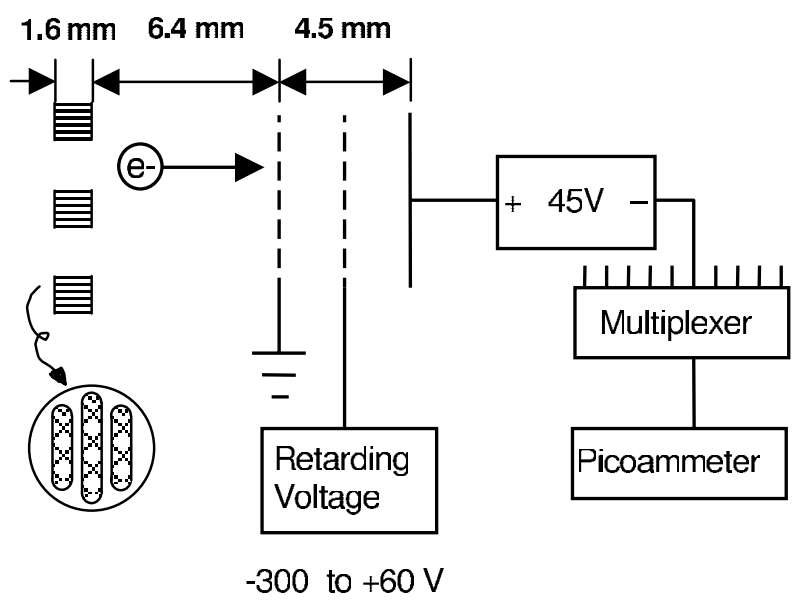

FIG. 3. Schematic of APS planar retarding field analyzer (RFA). The collector is graphite coated to lower $\delta$ and biased at $+45 \mathrm{~V}$ to enhance the collection efficiency. The grounded, outer grid is mounted behind a slotted vacuum penetration to shield the RFA from beam-induced rf noise. The penetration slot area is approximately $1.25 \mathrm{~cm}^{2}$, and approximate distances between the chamber wall (1.6 $\mathrm{mm}$ thick), grids, and collector are indicated. the fan is calculated for photons with energy above the photoelectron cutoff energy, using the expression for the synchrotron radiation vertical opening angle, $\Psi=$ $(1 / \gamma)\left(\varepsilon_{c} / \varepsilon\right)^{1 / 3}$. Here $\gamma$ is the beam relativistic factor, $\varepsilon \approx$ $4 \mathrm{eV}$ is the photoelectric work function, and $\varepsilon_{c}=$ $19.5 \mathrm{keV}$ is the critical photon energy. The horizontal limit of the fan is computed using the upstream photon absorber aperture and radiation opening angle defined by the bending magnet radius.

The RFA is an integrating device that transmits electrons with energy greater than the retarding grid voltage. In principle, the differentiated collector current is proportional to the electron energy spectrum. The detector response, however, shows a dependence on the angular distribution of the electrons. In Fig. 5(a), the measured response is shown for monoenergetic electron beams directed along the axis of the analyzer for energies of 53 and 105 eV. In Fig. 5(b), monoenergetic electrons (365, $1000 \mathrm{eV}$ ) are scattered from an $\mathrm{Al}$ target at $30^{\circ}$ from normal. The inset shows the differentiated signal of the $365-\mathrm{eV}$ beam near the transmission threshold. The measured detector response to scattered electrons resembles

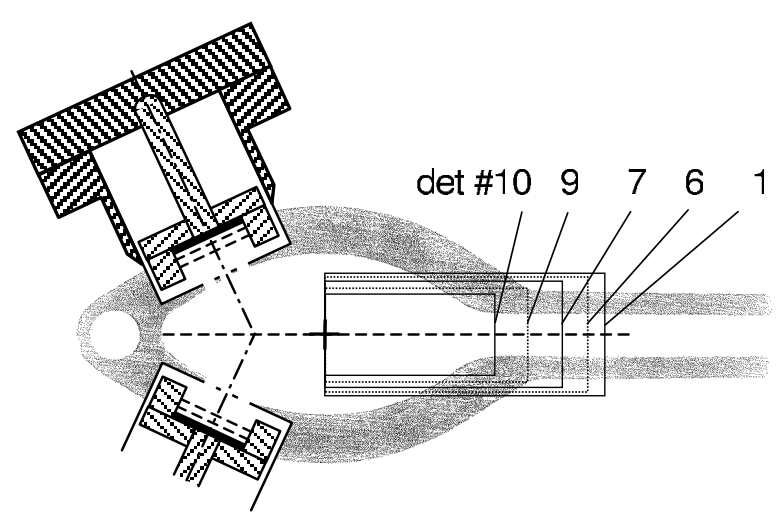

FIG. 4. Cross-sectional schematic view of the vacuum chamber showing mounted RFA detectors. The measured transmission through the two grids is $\sim 80 \%$. The synchrotron radiation fan cross sections for photons with energies $>4 \mathrm{eV}$ are shown schematically at selected detector locations. At detectors 3, 6, and 9, a standard BPM is mounted on top. The chamber dimensions, half width $\times$ half height, are $(42.5 \times 21) \mathrm{mm}$. 

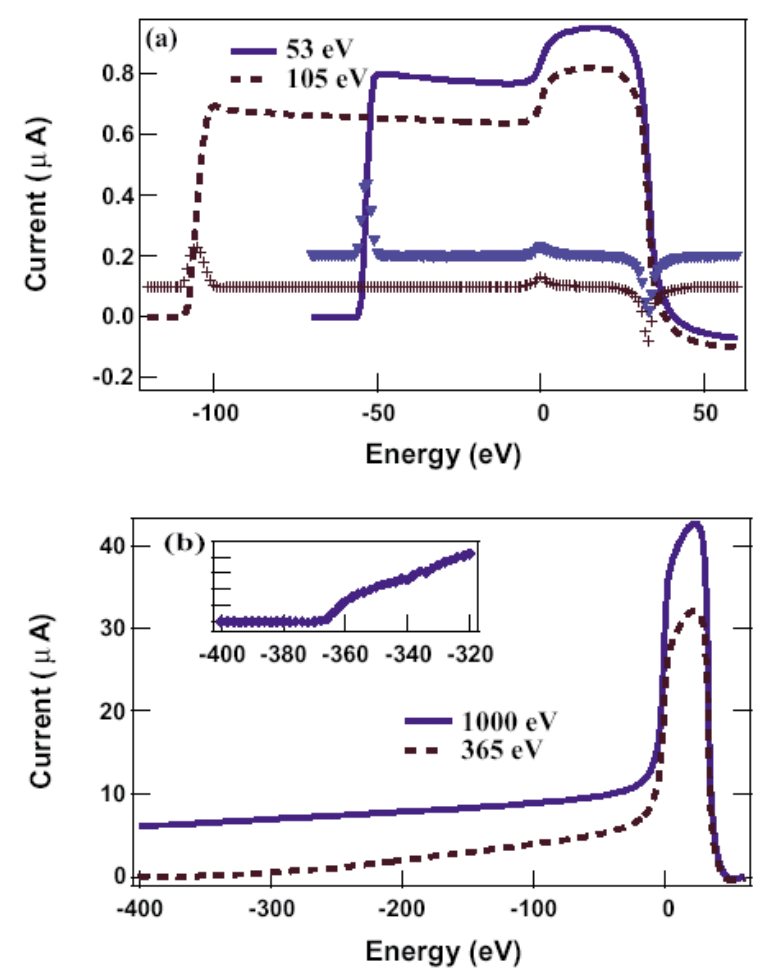

FIG. 5. (Color) (a) Transmission curves for the idealized case of monoenergetic electrons with perpendicular incidence angle. The differentiated signals are also shown. (b) Transmission curves for the more realistic case of monoenergetic electrons scattered from an Al target. The inset shows the differentiated signal for the $365-\mathrm{eV}$ beam near the transmission threshold.

more closely the situation we expect to encounter in the chamber.

In all the measurements, the collector is positively biased $(+45 \mathrm{~V})$ to "focus" the electrons and thereby increase the collection efficiency of the device. The effect can be seen in the bench measurements in Fig. 5. The maximum collector current occurs with about $+35 \mathrm{~V}$ bias, giving a value $\sim 20 \%$ higher than at zero bias. Above $+35 \mathrm{~V}$, the collector signal decreases as higherenergy electrons are no longer focused or are accelerated away from the collector. The RFA grid transmission for normal incidence was measured (at the maximum collector signal) to be 0.9 per grid, giving 0.8 for two grids. These transmission data are close to theoretical expectations for monoenergetic electron beams with small angular divergence. In practice, the RFA is less efficient than ideal; therefore the true wall current can be underestimated. Other possible contributions to the error in the absolute collector current include secondary electrons produced by collisions with the grids, the walls of the slots, and the inside walls of the RFA housing. Secondary electron emission from the collector surface is minimized by the graphite coating.

One of the advantages of the RFA design is that the cloud electrons in the chamber are shielded from the field

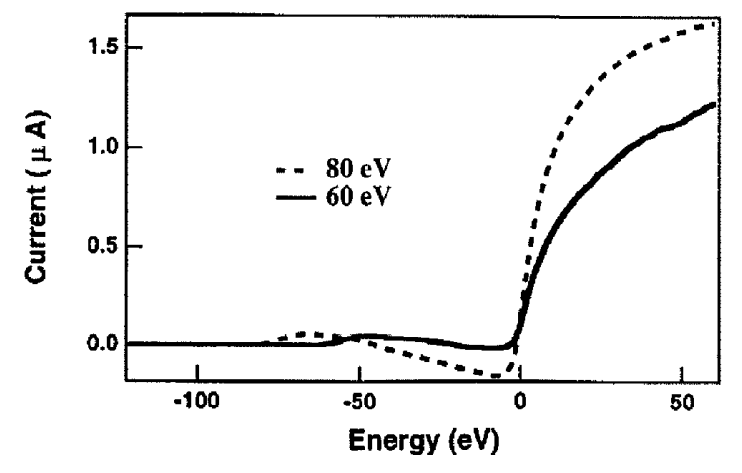

FIG. 6. Signal produced from a BPM irradiated by $60-\mathrm{eV}$ and $80-\mathrm{eV}$ electrons as a function of bias voltage applied to the BPM [13]. Similar signals were observed with the BPMs installed in the ring during the electron-cloud experiments.

induced by the retarding grid potential; it is a nonperturbative diagnostic. The difficulties of extracting the electron distributions using biased BPMs or clearing electrodes is illustrated in Fig. 6. Varying the bias voltage changes the electron collision energy. This changes the secondary emission from the BPM surface since $\delta$ is a function of the incident electron energy - the energy dependence of $\delta$ is well fit experimentally by a universal curve, shown in Fig. 7 [22,23]. In Fig. 6, note that the collected current can change sign; in this case more electrons are being emitted than collected. Finally, the collection length also changes with the bias voltage; this is seen in the growing collected current as the voltage becomes increasingly positive. These effects together make it very difficult to deduce the true wall flux with a biased BPM.

RFA and BPM data were multiplexed and collected using a PC-based data acquisition program. In a typical

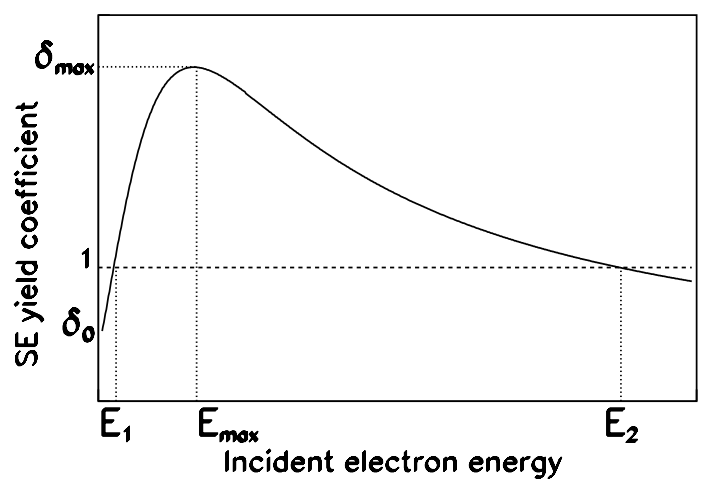

FIG. 7. Universal secondary electron yield coefficient curve $\delta(E)$, where $E$ is the incident electron energy. The maximum value of $\delta$ occurs at $\delta_{\max }$ for an incident energy $E_{\max }$. The value of $\delta$ is greater than unity in the range $E_{1}<E<E_{2}$, and $\delta_{0}$ is the finite probability that very low-energy electrons are reflected. The curve depends on the surface material, surface conditioning, and incident angle. Typical values are $\delta_{\max } \approx$ $1-3, \delta_{0} \approx 0.5, E_{\max } \approx 300 \mathrm{eV}, E_{1} \approx 50 \mathrm{eV}$, and $E_{2} \approx 1 \mathrm{keV}$. 
data scan, the retarding voltage was varied from $-300 \mathrm{~V}$ to $+60 \mathrm{~V}$ while the time-averaged collector current was acquired using a picoammeter. The collector current ranged from about $1 \mathrm{pA}$ to $1 \mu \mathrm{A}$, depending on machine parameters.

\section{ELECTRON-CLOUD STUDIES}

At the APS, amplification of the electron cloud due to secondary production is expected to lead to the most serious EC effects. Studies at the APS storage ring were designed to characterize and distinguish among the various contributions to the electron cloud. Typically, bunch currents between 1 and $3 \mathrm{~mA}$ ( 3.7 and $11 \mathrm{nC}$, respectively) were stored in up to 50 total bunches that were spaced varying between $2.8 \mathrm{~ns}\left(1 \lambda_{\mathrm{rf}}\right)$ and $0.36 \mu \mathrm{s}\left(128 \lambda_{\mathrm{rf}}\right)$. The total current was limited to $100 \mathrm{~mA}$.

For a fixed beam energy, the average total number of primary electrons is expected to be linear with beam current and independent of the temporal distribution of the beam. This contribution includes photoelectrons and secondaries produced in the collision of the photons with the walls [24]. In the absence of multipacting effects, the local electron density will depend primarily on the distance from the main electron source EA, and in a minor way on electrons produced by the bending magnet radiation and by fluorescence $x$ rays emitted from EA in the upstream direction to the beam.

In contrast, the total number and energy distribution of SEs, produced in collisions with the walls by EC electrons accelerated by the beam will be highly dependent on the bunch charge and spacing. While the secondaries emitted from the chamber surface have an intrinsic energy distribution, acceleration by the beam increases the average collision energy of the electrons. Because $\delta$ is energy dependent, the beam-cloud interaction can strongly influence the electron gain. Furthermore, the bunch spacing can satisfy a resonance condition known as BIM. This will be discussed in more detail in the next section.

Finally, measurements were compared before and after venting the test chamber to air, and after beam scrubbing to measure the effect on the cloud.

\section{POSITRON BEAM}

A representative set of RFA data is shown in Fig. 8 for detector 6. In (a) in the figure, the collector current $I_{c}$, normalized to the total beam current $I_{b}$, is shown as a function of the retarding voltage $V$ for ten bunches as a function of bunch spacing. In this example, the bunch current is fixed at $2 \mathrm{~mA} /$ bunch. The enhancement of the signal for a $7-\lambda_{\mathrm{rf}}$ bunch spacing is clearly evident. These are the integrated electron energy spectra; for negative bias, electrons with energy greater than $e \times V$ are transmitted to the collector, where $e$ is the electron charge. The
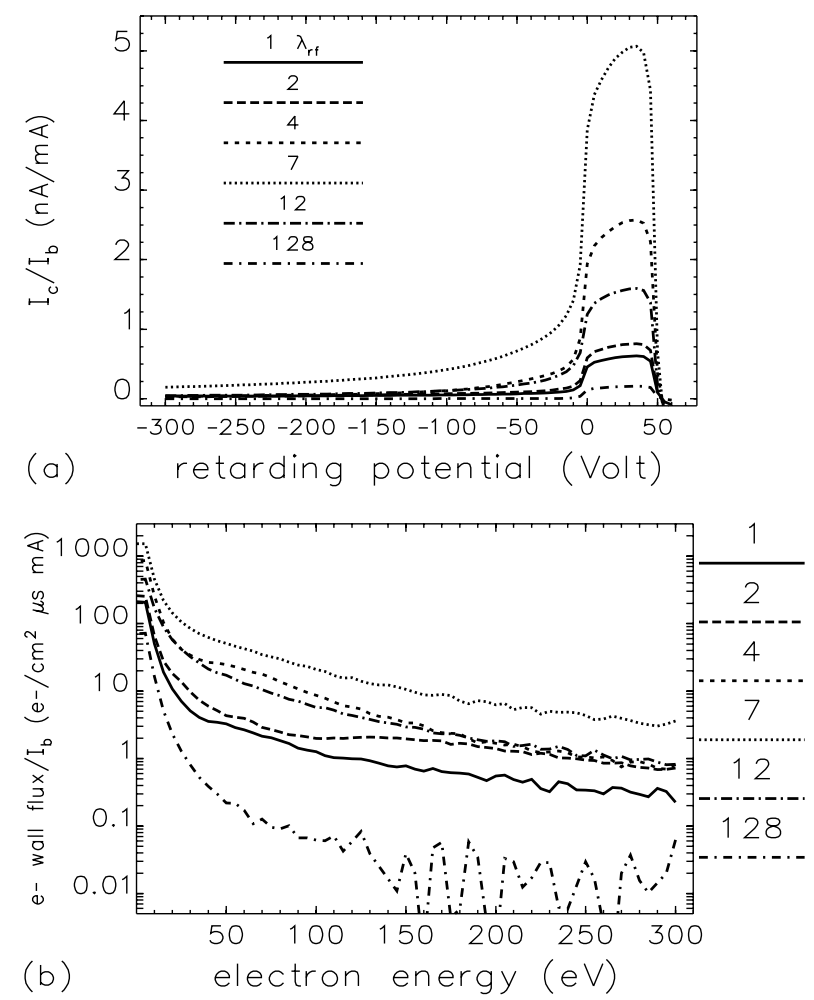

FIG. 8. (a) Normalized collector current versus retarding potential, $e+$ beam, $20 \mathrm{~mA}$, ten bunches, as a function of bunch spacing, in units of $\lambda_{\mathrm{rf}}=2.84 \mathrm{~ns}$. (b) Electron energy distributions obtained by differentiating the data in (a). The data show that the cloud has the longest energy tail at the beam-induced multipacting bunch spacing of $20 \mathrm{~ns}\left(7 \lambda_{\mathrm{rf}}\right)$. The low-energy parts of the distributions (and $128 \lambda_{\mathrm{rf}}$ spacing case) are well fitted by a Lorentzian function of width $4 \mathrm{eV}$ and mean energy $2.5 \mathrm{eV}$. The high-energy part is exponential.

signal rises sharply near zero bias, an indication that most of the electrons striking the wall have low energy.

The RFA data are differentiated with respect to the bias, $V$ in Fig. 8(b), giving the electron energy spectra. It should be noted that these spectra are convolved with the detector response, where the incident electron angular distribution is unknown; this is similar to the case in Fig. 5(b). The net result is a loss in energy resolution; however, one can compare the general features of the spectra. In all cases, the low-energy parts of the distributions are well fit with a Lorentzian function $(\Gamma / 2) /\left[(\Gamma / 2)^{2}+(E-\langle E\rangle)^{2}\right]$, where the width $\Gamma$ is $4 \mathrm{eV}$, and mean energy $\langle E\rangle$ is $2.5 \mathrm{eV}$. The high-energy part results from electrons accelerated by the beam and is observed to fall off exponentially. For the longest spacing $\left(128 \lambda_{\mathrm{rf}}\right)$, there is virtually no exponential tail; we can assume that most of the cloud electrons have been lost before the next bunch passage. The normalized electron current at this large spacing is virtually the same as for a single bunch, further supporting this conclusion. For bunch spacings at the BIM resonance $\left(\begin{array}{ll}7 & \lambda_{\mathrm{rf}}\end{array}\right)$, the 


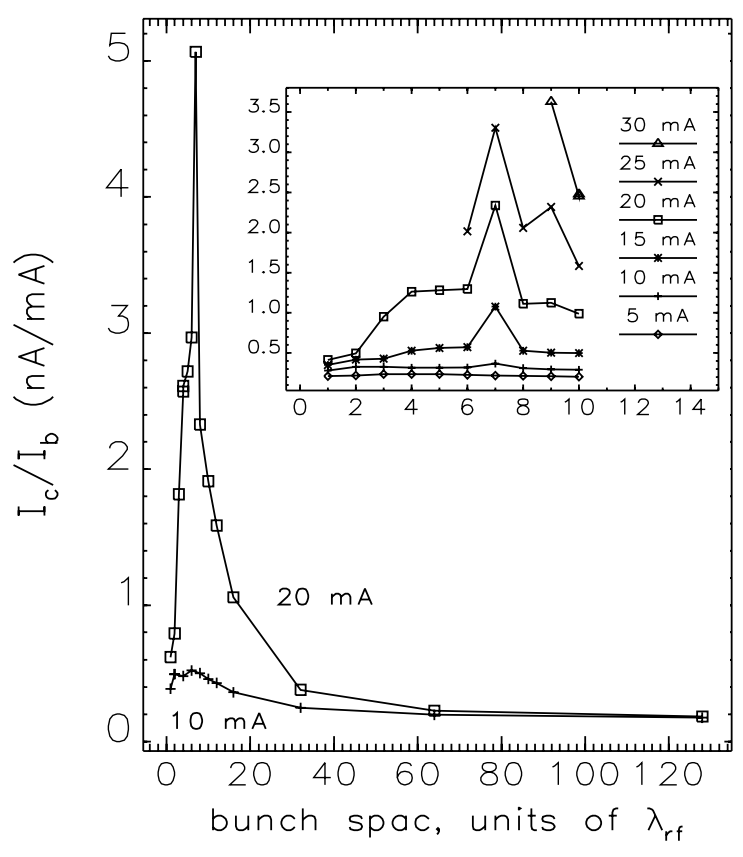

FIG. 9. Comparison of normalized electron current as a function of bunch spacing and bunch current (ten bunches, total beam current shown; detector 6). Vacuum pressure rise and sharp peak at $7 \lambda_{\text {rf }}$ only observed for bunch currents above about $1.5 \mathrm{~mA}$. Figure inset shows detail around the peak and also shows the effect of surface conditioning, having been acquired after an additional $60 \mathrm{Ah}$ of beam operation.

exponential tail is the longest. Additional features can be seen on the tail, e.g., at $2 \lambda_{\mathrm{rf}}$ and $4 \lambda_{\mathrm{rf}}$.

The beam-induced multipacting resonance is most clearly seen in a plot of the maximum RFA collector signal (positive bias) versus the bunch spacing; this is shown in Fig. 9. In the main plot, the normalized collector current is shown for 10 and $20 \mathrm{~mA}$ total beam current. There is both a broad peak and a very sharp peak, both centered at $7 \lambda_{\text {rf }}$. This is more evident in the inset in the figure, which shows more detail around the peak. The position of the peak does not vary with bunch current, as one might expect considering the difference in the electron energy gain (note in the main plot, the data point for $7 \lambda_{\mathrm{rf}}$ is absent in the $10 \mathrm{~mA}$ data). It is interesting to note that the signal levels in the inset are reduced by about a factor of 2. These data were acquired after about $60 \mathrm{Amp}-\mathrm{h}(\mathrm{Ah})$ of beam operation compared with the main plot. More will be said about this conditioning effect later.

\section{A. Beam-induced multipacting, including SE energy}

In its simplest form, BIM is a resonance condition in which cold electrons at the wall are accelerated by the beam and traverse the chamber in precisely the time between bunch passages $t_{b}$. On striking the wall, secondary electrons are produced (assumed with zero energy), which are again accelerated to an energy $E$ by the beam.
If $\delta(E)$ is greater than unity, this process can lead to amplification of the electrons. In the impulse approximation, valid for short bunches, the energy gain $\Delta K$ is given by [16]

$$
\Delta K=2 m_{e}\left(c r_{e} N_{b} / r\right)^{2},
$$

where $m_{e}$ is the electron mass, $c$ is the speed of light, $r_{e}=$ $2.81785 \times 10^{-13} \mathrm{~cm}$ is the classical electron radius, $N_{b}$ is the bunch population, and $r$ is the half-chamber radius. Assuming the accelerated electron travels a distance $2 r$, the resonance condition is then given by [16]

$$
t_{b}=\frac{2 r}{\sqrt{2 \Delta K / m_{e}}}=\frac{r^{2}}{c r_{e} N_{b}} .
$$

We can assume that the electrons undergoing the resonance are constrained to travel in a path that crosses the center of the chamber and that they strike the chamber walls at nearly normal incidence. We also assume that off resonance, the electrons strike the wall and are not accelerated immediately by the beam. Secondary electrons produced at the wall are emitted with a $\cos \theta$ angular distribution [23] and drift according to their emitted energy distribution.

Applying the BIM resonance condition to the APS in the case with $2 \mathrm{~mA} /$ bunch $\left(N_{b}=4.6 \times 10^{10}\right)$, using values of $r$ ranging between the two chamber semiaxes (see Table I), we obtain bunch spacings corresponding to between 4 and $16 \lambda_{\text {rf }}$ [25]. This result is consistent with the amplification of electrons giving rise to the broad peak in Fig. 9; however, the sharp peak at $7 \lambda_{\mathrm{rf}}$ is not predicted by this simple formula.

The sharp peak can be partly explained by the RFA detector position on the chamber and by the transmission efficiency of the device. At the mounted location of the RFA, the half-chamber radius $r$ ranges from about 2.6 to $3.2 \mathrm{~cm}$ over the width of the RFA aperture. The angular acceptance for electrons traveling along a chamber radius is about $20^{\circ}$ to $40^{\circ}$ from the detector normal. Using $2 \mathrm{~mA}$ per bunch and Eq. (2), the BIM resonance is satisfied for $17 \leq t_{b} \leq 23 \mathrm{~ns}\left(6\right.$ to $\left.8 \lambda_{\mathrm{rf}}\right)$. The transmission for normal incidence through the two RFA grids was measured to be 0.8. In addition, the transmission through the vacuum chamber penetration behind which the device is mounted was estimated assuming a 1/16-in. slot wall thickness and a 1/8-in. slot width. Off the BIM resonance, the RFA acceptance for electrons scattered from the wall is about $\pm 60^{\circ}$. We assume a $\cos \theta$ incident electron angular distribution [23] and integrate over a slot. The result is an average transmission of 0.6 , giving a total device transmission of $(0.8)(0.6)=0.5$. On resonance, the incidence is almost normal, and the total transmission is nearly 0.8 . The net result is that we expect $\sim 50 \%$ detector efficiency over the full range of bunch spacings except near 6-8 $\lambda_{\text {rf }}$, where we expect $\sim 80 \%$ efficiency. 
The detector transmission efficiency does not explain all the data, however. The position of the sharp peak is not very sensitive to the bunch current (see the inset in Fig. 9). Using $2.5 \mathrm{~mA}$ per bunch satisfies the BIM resonance for $14 \leq t_{b} \leq 19$ ns ( 5 to $7 \lambda_{\mathrm{rf}}$ ), while $3 \mathrm{~mA}$ gives $12 \leq t_{b} \leq$ $15 \mathrm{~ns}$ ( 4 to $5 \lambda_{\mathrm{rf}}$ ). This implies that the sharp peak should shift, which is not observed. These data suggest that a more general BIM condition also depends on the SE energy distribution, which consists of three components: true secondaries, elastically scattered, and inelastically scattered electrons [26]. The distribution of the true secondaries peaks around $1-3 \mathrm{eV}$, independent of the electron collision energy and, therefore, the beam current. In this scenario, first suggested by Furman and Heifets [27], lowenergy secondary electrons produced between bunch passages drift near the beam at the chamber center. The electrons are accelerated to high energies [i.e., near the maximum of $\delta(E)$ vs $E$ curve, see Fig. 7], striking the walls and producing secondary electrons with high probability. In the time before the next bunch passage, the low-energy secondaries once again drift near the beam. The general form of the BIM resonance can be written as

$$
t_{b}=t_{\mathrm{SE}}+t_{a}=\frac{(r-a)}{\sqrt{2 K_{\mathrm{SE}} / m_{e}}}+\frac{a(r \pm a)}{2 c r_{e} N_{b}},
$$

where $a$ is the distance between the secondary electron and the beam at the time of the bunch passage, $t_{\mathrm{SE}}$ is the drift time of the secondary from the wall to distance $a, t_{a}$ is the drift time of the secondary from distance $a$ to the opposite wall after it is accelerated by the beam, and $K_{\mathrm{SE}}$ is the secondary electron emitted energy. The last term is the same as Eq. (2), where $\Delta K$ is given by Eq. (1), where distance $r$ is replaced by $a$, and distance $2 r$ has been replaced by $(r \pm a)$, where the top sign corresponds to the case with a positron beam and the bottom sign to the case with an electron beam. A preliminary calculation assuming a positron bunch spacing of $7 \lambda_{\mathrm{rf}}$ and assuming that the most probable secondary electron emitted energy is 1 to $2 \mathrm{eV}$ [26] suggests that this resonance is indeed satisfied for an electron starting at a distance of about $1 \mathrm{~cm}$ from the beam. For bunch currents from 1.5 to $2.5 \mathrm{~mA}$ and a range of values of $r$, the electron energy gain ranges from about 100 to about $300 \mathrm{eV}$, respectively. Interestingly, this energy range corresponds to the range of incident energies where, typically, $\delta>1$ (cf. Fig. 7). A more detailed calculation is being undertaken to apply this general form of the BIM resonance to all the experimental data more systematically.

Several observations confirm the important role of lowenergy secondaries in the BIM condition. We have already seen in Fig. 8(b) that the energy tail at $7 \lambda_{\mathrm{rf}}$ is the longest, confirming that electrons are in fact drifting near the beam and getting accelerated to energies of the order of $100 \mathrm{eV}$ at the BIM resonance. A closer look at the features in the energy distribution for 2- or $4-\lambda_{\mathrm{rf}}$ bunch spacing also suggests that there is a resonance condition that "selects" electrons at a certain distance from the beam at each bunch passage. The electrons collide with the wall with the corresponding energy, which results in an enhanced signal at a given energy. Finally, in modeling the APS experiments using the code POSINST, developed by Furman and Pivi at Lawrence Berkeley National Laboratory, the position and width of the broad peak in the electron-cloud flux versus bunch spacing curve was indeed found to be sensitive to the secondary electron distribution [25,28].

\section{B. Electron-cloud buildup and saturation}

Before discussing the electron-cloud buildup and saturation, it should be noted that significant variation was seen from one detector to another, especially for BIM conditions [14]. The gain in the detector signals as a function of bunch spacing or number of bunches varied according to location. Detectors near the absorber EA typically exhibited the smallest gains (factors of 2 to 3 ), while detectors farther upstream (e.g., detectors 6-9) exhibited gains of over a factor of 100. The effect of EA as a local source of electrons appears to dominate in the detectors nearby. Farther from EA, the effect of multipacting is more clearly observed.

The longitudinal variation of the cloud is illustrated in Fig. 10, in which the RFA signals are plotted as a function of distance from EA. Ten bunches are shown with either $128-\lambda_{\mathrm{rf}} 10(\mathrm{c})$ or $7-\lambda_{\mathrm{rf}} 10$ (b) bunch spacing, and at $7-\lambda_{\mathrm{rf}}$ spacing, 50 bunches are also shown 10(a). The bunch current is $2 \mathrm{~mA}$ for all cases. The photoelectron current is estimated assuming an antechamber, using the radiation fans at the detector locations, and is scaled to the 128- $\lambda_{\mathrm{rf}}$ data. The detector current for $128-\lambda_{\mathrm{rf}}$ spacing roughly follows the predicted photocurrent except at detectors 1-2, where it deviates sharply. In this case, the influence of primary electrons produced at EA on the cloud distribution nearby (upstream) can explain the enhanced signals at detectors $1-2$. With a $7-\lambda_{\text {rf }}$ spacing (ten bunches), the effect of EA is no longer a simple function of distance; the signals at detectors 6-9 show more amplification than at detectors $1-3$. With 50 bunches, the effect is larger still: the electron signals at detectors 6-9 exceed those at detectors $1-3$. At detector 1 , the overall amplification from (c) to (a) in Fig. 10 is 2.4, whereas at detector 9, it is 150 . This result cannot be explained by the primary source of electrons, which is 50 times smaller at detector 9 than at detector 1 , as measured with one bunch. Possibly, the large source of primary electrons near EA interferes with the BIM process by injecting electrons with the wrong energy during the bunch passage. Multiple photon reflections or fluorescence $\mathrm{x}$ rays from EA may produce photoelectrons after the bunch passes. This process can inject a source of 


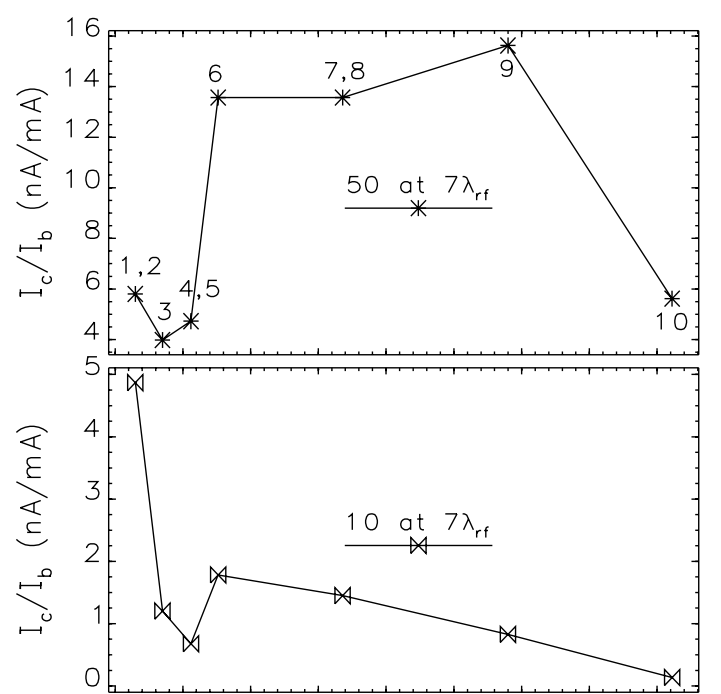

(a)

(b)

(c)

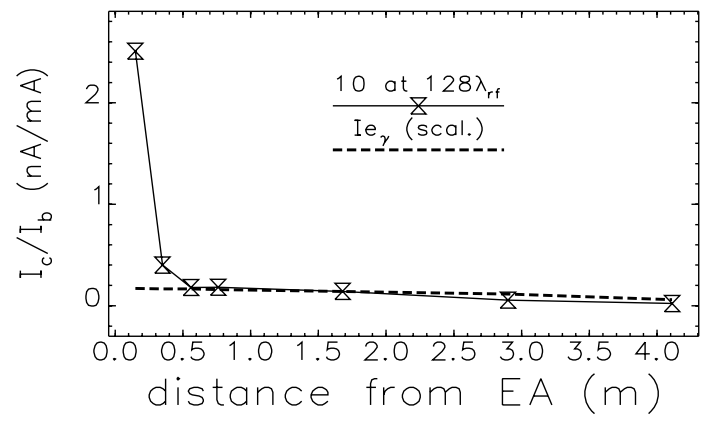

FIG. 10. Total, normalized electron current per detector as a function of distance from EA for (a) 50 bunches at 7- $\lambda_{\mathrm{rf}}$ bunch spacing (detector locations are marked); (b) ten bunches at $7-\lambda_{\text {rf }}$ bunch spacing; and (c) ten bunches at $128-\lambda_{\text {rf }}$ bunch spacing. The beam intensity is $2 \mathrm{~mA}$ per bunch. In (c), the calculated photoelectron current $I e_{\gamma}$ was estimated, using the radiation fans at the detector locations, and scaled to the $128-\lambda_{\mathrm{rf}}$ data. The normalized signals for 10 or 50 positron bunches spaced at $7 \lambda_{\text {rf }}$ show a marked longitudinal dependence compared with case (c).

electrons that can drift near the beam between bunches and more efficiently seed the BIM resonance near the detectors upstream. The dip observed at detectors 3-5 is not clearly understood.

Measurements of the electron-cloud buildup and saturation over a bunch train are shown in Fig. 11. In 11(a), the normalized wall current is plotted as a function of bunches in the train: the bunch spacing is fixed at the BIM condition $\left(7 \lambda_{\mathrm{rf}}\right)$, and the bunch current is fixed at $2 \mathrm{~mA}$. The vacuum pressure $P$ measured near detector 9 (located $3 \mathrm{~m}$ upstream from EA) is also plotted. Again, the variation in detector location can be seen. In all cases, the wall flux increases exponentially and saturates (increases linearly) after a certain number of bunches. The rate of the exponential rise, the number of bunches after which the cloud saturates, and saturation level at $100 \mathrm{~mA}$ (total current) vary by detector location. The saturation levels vary by a factor of 3 at the detectors shown. At

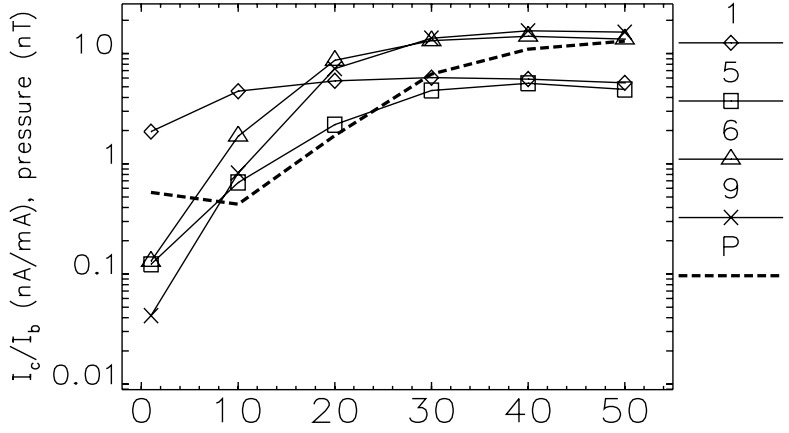

(a) number of bunches in train, $\mathrm{N}_{\mathrm{b}}$

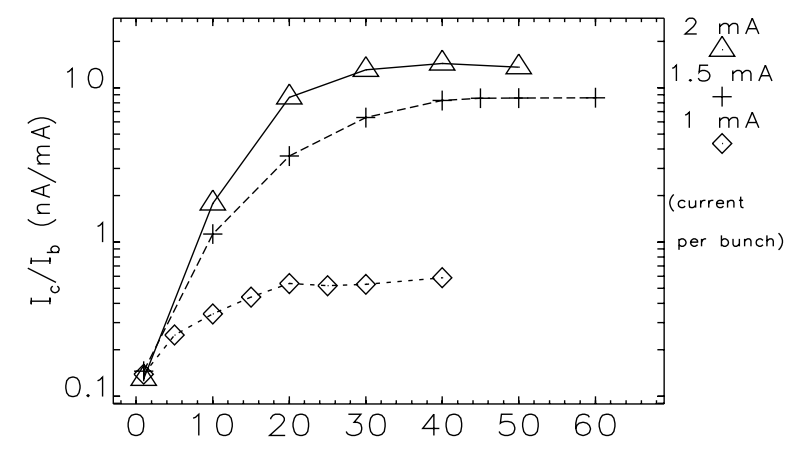

(b) number of bunches in train, $N_{b}$

FIG. 11. Growth and saturation of electron cloud over a train of positron bunches, spaced at $7 \lambda_{\text {rf }}$. In (a), the data are shown as a function of detector number (detectors 1, 5, 6, and 9 with $2 \mathrm{~mA} /$ bunch). The vacuum pressure $P$, measured locally, is also shown. The vacuum pressure at $100 \mathrm{~mA}$ for nominal (non$\mathrm{BIM}$ ) operating conditions is $0.5 \mathrm{nT}$, to be compared with a value of $10 \mathrm{nT}$ for 50 bunches (100 mA) at BIM conditions. The bunch train length at which the cloud saturates varies from 10 to 30 bunches, and the electron-cloud saturation level varies by up to a factor of 5. In (b), detector 6 is shown as a function of bunch current; the saturation level varies nonlinearly with bunch current.

detector 1 , the normalized signal is almost at saturation with two bunches, growing by only a factor of 2 after 20 bunches. At detector 9, the normalized signal grows by a factor of 350 after 30 bunches. Figure 11(b) shows the cloud buildup at detector 6 when the bunch current is varied. The cloud reaches saturation in all cases after about 20 bunches, and the saturation levels vary with bunch current in a nonlinear way.

\section{Electron-cloud-induced beam instability}

A preliminary analysis of turn-by-turn BPM data acquired during the final run with positrons shows that a horizontal coupled-bunch instability occurs for a bunch spacing of $7 \lambda_{\text {rf }}(20 \mathrm{~ns})$ and $\sim 2 \mathrm{~mA} /$ bunch; i.e., the BIM conditions. Figure 12 shows the horizontal bunch centroid offset for each of 50 bunches, $90 \mathrm{~mA}$ total. Five consecutive turns are shown (the horizontal tune is 0.2). This instability is not observed with electron beams for 


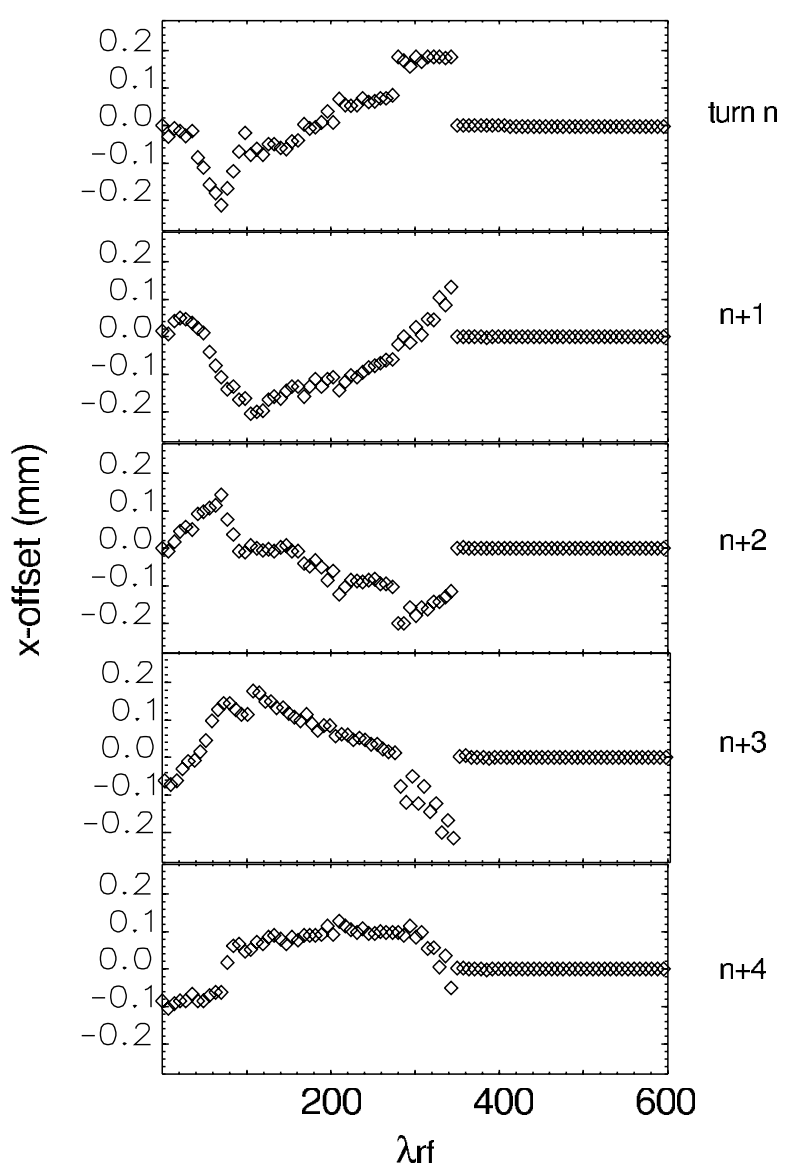

FIG. 12. Bunch-by-bunch horizontal centroid oscillations using turn-by-turn BPM data acquisition for positron beam (50 bunches, $90 \mathrm{~mA}$ total, 7- $\lambda_{\text {rf }}$ BIM spacing). The head of the train is on the left.

otherwise identical conditions. Analyses of these data are ongoing.

\section{ELECTRON BEAM}

One of the more interesting results with electron beams is that amplification of the electron cloud does occur, albeit at a more modest level compared to positron beams. The measured RFA data for ten electron bunches with $2 \mathrm{~mA} /$ bunch whose spacing is varied in shown in Fig. 13(a). The maximum signal occurs for $11 \lambda_{\text {rf }}$. That the bunch spacing for maximum amplification is different with electron beams is not surprising since the dynamics of the beam-cloud interaction is governed by the sign of the acceleration; the general BIM condition is now given by the lower sign in Eq. (3). Unlike with positrons, however, no sharp peak is observed, only a broad peak. The buildup and saturation of the cloud is shown at different detectors in Fig. 13(b). With electron beams, the detectors nearest EA show the highest signal levels, and the vacuum pressure rise for 50 bunches at $11 \lambda_{\mathrm{rf}}$ bunch spacing more closely agrees with the nominal pressure of $0.5 \mathrm{nT}$ for a $100-\mathrm{mA}$ user beam.
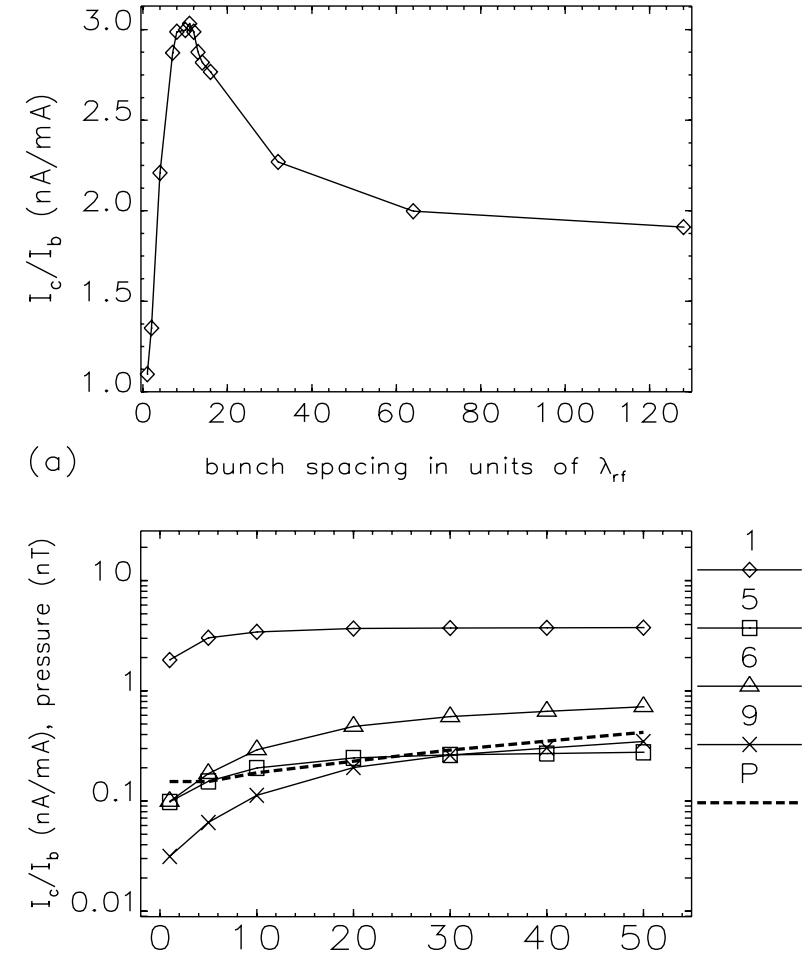

(b) number of bunches in train, $\mathrm{N}_{\mathrm{b}}$

FIG. 13. (a) Detector 1 for ten bunches as a function of bunch spacing. (b) Growth and saturation of electron cloud over the train of electron bunches, spaced at $11 \lambda_{\mathrm{rf}}$, as a function of the detector number. The local pressure $P$ is also shown. In all cases, the bunch current is $2 \mathrm{~mA}$. There is an additional surface conditioning of $100 \mathrm{Ah}$ for these data, compared with Fig. 11.

The absence of the sharp resonance suggests the following explanation of the difference between positron and electron beams. In preliminary calculations applying Eq. (3), it appears that the general BIM condition is satisfied for electrons that drift very close to the beam, then accelerate to energies in the $\mathrm{keV}$ range. When these electrons collide with the walls, the amplification is low since $\delta$ is small, and the resonance is not sustained. An examination of the energy spectra, shown in Fig. 14, shows that high-energy electrons are indeed not observed. On average, for the same bunch current, the energy of the electrons striking the walls is lower for electron beams than for positron beams. It appears that with electron beams, low-energy secondary electrons created at the chamber wall cannot drift as closely to the beam center between bunch passages and are instead driven back into the wall almost as soon as they are created. With an average measured energy of only $10 \mathrm{eV}(2 \mathrm{~mA} /$ bunch, $11-\lambda_{\mathrm{rf}}$ bunch spacing), this implies that the electrons on average drift to about $4 \mathrm{~cm}$ from the beam [Eq. (1)]; this is essentially the starting distance for photoelectrons above and below the antechamber slot. In contrast, with positron beams with $2 \mathrm{~mA} /$ bunch, the average energy is $100 \mathrm{eV}$, implying an average approach of about $1.3 \mathrm{~cm}$. 


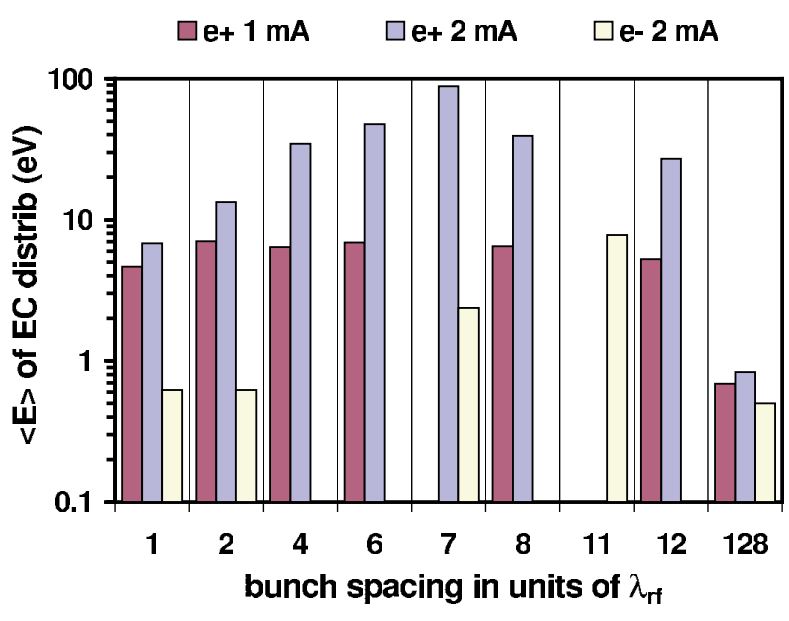

FIG. 14. (Color) Average wall collision energy calculated from measured electron-cloud energy distributions as a function of bunch spacing (ten bunches) for positron (1-mA and 2-mA bunch current) and electron (2-mA bunch current) beams. For these data, the highest average energy (100 eV) occurs for positron beams with $2 \mathrm{~mA} /$ bunch and $7-\lambda_{\text {rf }}$ bunch spacing. In comparison, the highest average energy for electron beams with $2-\mathrm{mA}$ bunches is about $10 \mathrm{eV}$, occurring for $11-\lambda_{\mathrm{rf}}$ bunch spacing. The lower average energy suggests that the cloud electrons are not getting as close to the beam with an electron beam compared with a positron beam. The average energy with $1-\mathrm{mA}$ positron bunches is less than $10 \mathrm{eV}$. There is an additional surface conditioning of $100 \mathrm{Ah}$ for the electron beam data.

Incidentally, the average electron energy for positron beams with $1 \mathrm{~mA} /$ bunch is less than $10 \mathrm{eV}$, and little or no sharp peak is observed (Fig. 9, inset). We conclude that in this case as well, the general BIM condition is not sustained.

Despite the differences between positron and electron beams, Figs. 11(a) and 13(b) show that near EA (e.g., detector 1) the cloud buildup and saturation are within a factor of 2 for 2-mA positron or electron bunches. Since the electron beam data were acquired after $100 \mathrm{Ah}$ of beam operation, surface conditioning may largely explain the difference. On the other hand, it is mainly the energy spectra and electron-cloud buildup far from the EA that distinguish the results for positron versus electron beams. Analysis of the spectra thus provides a better measure of the beam-cloud interaction than simply the wall flux.

To date, the only indication of cloud-induced effects in electron beams was a decrease in the beam lifetime that occurred at certain bunch fill patterns. For example, we filled $85 \mathrm{~mA}$ in nine bunch trains of four bunches each. The vacuum pressure was a factor of 2 higher and the beam lifetime about half with a $2-\lambda_{\text {rf }}$ gap between bunch trains, compared to a gap of $12 \lambda_{\mathrm{rf}}$. The RFA signals were a factor of 3 to 5 higher with the smaller gap. These observations are consistent with electron-stimulated gas desorption. When these machine conditions were repeated after additional surface conditioning, the vacuum pressure rise was reduced considerably.
At the largest observed EC amplification, using single bunches spaced at $11 \lambda_{\mathrm{rf}}$, no cloud-induced effects have been observed up to $2 \mathrm{~mA} /$ bunch. For electron beams, we postulate that electron-stimulated gas desorption may indirectly lead to beam-ion instabilities if a sufficient number of desorbed gas molecules become ionized by the beam.

\section{SURFACE CONDITIONING}

By repeating measurements over a period of time, before and after the test chamber was vented to air, we had an opportunity to observe the effects of surface conditioning by the beam, sometimes referred to as beam scrubbing.

When the new test chamber was first installed, standard bakeout procedures were followed: (135 C for $24 \mathrm{~h}$; ramp temperature up/down at a rate of $10 \mathrm{C} / \mathrm{h}$ ). After 75.5 Ah of accumulated beam operation, the chamber was vented to air. The RFA data shown in the main plot in Fig. 9 were acquired $0.8 \mathrm{Ah}$ after venting. The scan in bunch spacing was repeated $62 \mathrm{Ah}$ after venting, shown in the inset in Fig. 9. The peak RFA signal values at a bunch spacing of $7 \lambda_{\mathrm{rf}}$ are reduced by more than a factor of 2 for the same bunch current. The peak RFA signal values at a bunch spacing of $128 \lambda_{\mathrm{rf}}$ (not shown) were reduced by a smaller amount: about $30 \%$.

The approximate electron dose on the chamber surface between the two sets of data can be estimated by assuming that between measurements, $100 \mathrm{~mA}$ positron beam was stored with the standard user bunch pattern (81 bunches). Machine study periods where arbitrary bunch patterns had been used are not accounted for in this approximation. The dose at detector 6 is given by

$$
\left(I_{c} / I_{b}\right)_{\mathrm{std}} / A_{\mathrm{RFA}} \times 60 \mathrm{Ah}=10^{18} e / \mathrm{cm}^{2}=0.1 \mathrm{C} / \mathrm{cm}^{2},
$$

where $A_{\mathrm{RFA}}$ is the detector aperture, $\sim 1 \mathrm{~cm}^{2}$, and $\left(I_{c} / I_{b}\right)_{\text {std }}$ was measured to be $0.6 \mathrm{nA} / \mathrm{mA}$. These results are consistent with bench measurements that show a reduction in $\delta$ under electron bombardment [19]. The APS RFA data also indicate that the surface conditioning effect appears to depend on whether primary electrons (large bunch spacing) or secondary electrons (BIM resonant bunch spacing) dominate. The reduction in the electron cloud appears greater in the latter case.

Conditioning of the vacuum chamber surfaces by beam scrubbing is sensitive to the beam trajectory. This effect was observed by steering the beam locally; the beam trajectory had been kept constant at the RFA locations throughout all operation prior to the test. Shifting the orbit parallel to the original trajectory resulted in no change; however, after steering the beam vertically by $20 \mu \mathrm{rad}(0.5 \mathrm{~mm}$ displacement at the end of the $5-\mathrm{m}$ chamber), the RFA signal at detector 6 increased by a factor of 2. The beam trajectory in the upstream dipole was kept fixed. This test was done with an electron beam: $100 \mathrm{~mA}$ in 50 bunches spaced at $11 \lambda_{\mathrm{rf}}$. 


\section{ELECTRON-CLOUD DENSITY}

In proton rings, a convenient figure of merit is the ratio of the average electron-cloud density to the average beam density. For example, at the PSR, the electron-proton instability threshold occurs when this ratio is $\sim 1 \%$ [4]. From Fig. 11, the cloud density $n_{\mathrm{EC}}$ can be very roughly estimated in the APS given the measured wall flux and the average electron velocity. The average beam density $n_{\text {beam }}$ is calculated as if the beam were uniformly distributed in the chamber. For example, taking $100 \mathrm{~mA}$ total current, $2 \mathrm{~mA}$ per positron bunch, and $7 \lambda_{\mathrm{rf}}$ bunch spacing:

$$
n_{\mathrm{EC}}=I_{c} /\left(A_{\mathrm{RFA}} e\left\langle v_{e}\right\rangle\right)=10^{4} \mathrm{~cm}^{-3},
$$

and

$$
n_{\text {beam }}=N_{b} /\left(s_{b} A_{\mathrm{vc}}\right) \times F=10^{6} \mathrm{~cm}^{-3} .
$$

Here $\left\langle v_{e}\right\rangle$ is the velocity, $6 \times 10^{8} \mathrm{~cm} / \mathrm{s}$, of the averageenergy electron from the differentiated $d I_{c} / d V$ (Fig. 14); $s_{b}$ is the bunch separation in units of length; $A_{\mathrm{vc}}$ is the vacuum chamber cross-section area; and $F$ is the fill fraction, given by 50 bunches $\times s_{b}$ divided by the ring circumference. Saturation is observed at about $1 \%$ of the average beam density for 1.5 and $2 \mathrm{~mA} /$ bunch, and at only $\sim 0.1 \%$ for $1 \mathrm{~mA} /$ bunch. In addition, it appears that the coupled-bunch instability is observed only at saturation and only for positron bunch currents of no less than about $2 \mathrm{~mA} /$ bunch.

\section{SUMMARY}

Experiments have been carried out at the Advanced Photon Source storage ring using dedicated diagnostics to measure the properties of the electron cloud. The diagnostic is based on the planar retarding field analyzer, and both the time-averaged electron-cloud flux and energy spectrum were measured for electrons striking the vacuum chamber wall for varying machine conditions.

In the APS, amplification of the electron cloud was observed for certain bunch spacing. These data can best be explained by a more general expression for the BIM resonance condition that involves secondary electrons that drift close to the beam between bunch passages. For positron beams, a buildup of the electron cloud was observed to occur above a threshold bunch current of about $1.5 \mathrm{~mA} /$ bunch $(5.5 \mathrm{nC})$ with a bunch spacing of $7 \lambda_{\mathrm{rf}}(20 \mathrm{~ns})$. The electron cloud was observed to reach saturation at a level of about $1 \%$ of the average beam density after about 20-30 bunches at the BIM resonance. This observation indicates that the space charge of the cloud is sufficient to limit the otherwise exponential growth. A horizontal coupled-bunch instability was observed for positron bunch trains of 20 or more near the BIM resonant and with a threshold bunch current of about $2 \mathrm{~mA}(7.4 \mathrm{nC})$. Under these conditions, the vacuum pressure was $10 \mathrm{nT}$ at $100 \mathrm{~mA}$ (50 bunches), compared to the pressure of $0.5 \mathrm{nT}$ at $100 \mathrm{~mA}$ with nominal, nonBIM operating conditions. While a BIM resonance was observed with electron beams centered around an $11-\lambda_{\mathrm{rf}}$ bunch spacing ( $30 \mathrm{~ns}$ ), the amplification of the electron cloud is more modest than for positron beams. Coupledbunch instabilities have not been observed for electron beams with up to 50 bunches at $2 \mathrm{~mA} /$ bunch at either the $7-\lambda_{\mathrm{rf}}$ bunch spacing or the 11- $\lambda_{\mathrm{rf}}$ bunch spacing. However, vacuum effects consistent with electron-stimulated gas desorption have been observed, and these are correlated with electron-cloud amplification and degradation in beam lifetime. The possibility that electron-cloud effects can lead indirectly to beam-ion effects is under investigation.

Observations at the APS demonstrate the importance of including the general form of the BIM resonance in predictions of electron-cloud effects. The BIM resonance is sensitive to the details of the energy spectrum of the emitted secondary electrons; this parameter should, therefore, be rather important in simulations of the buildup of the electron cloud. The electron-cloud density is perhaps not as sensitive to the uncertainty in the peak secondary electron yield coefficient $\delta$ as it is to the uncertainty in the emitted secondary electron energy spectrum. The latter determines the conditions for the BIM resonance in the general case. Following beam conditioning, the RFA signal level was observed to decrease by about a factor of 2 , consistent with observations in the laboratory of surface conditioning under electron bombardment. A measurable longitudinal variation was observed in the electron cloud: the cloud saturation level varied by up to a factor of 5 measured at different locations in the ring. The signal levels at detectors more than $1 \mathrm{~m}$ upstream of a photon end absorber showed greater amplification than detectors near the absorber, even though the source of primary electrons was much higher for the latter. This observation suggests that geometrical details of the vacuum chamber giving rise to longitudinal effects should be accounted for in simulations.

\section{ACKNOWLEDGMENTS}

The authors gratefully thank J. Galayda for suggesting and encouraging this study; G. Goeppner, J. Gagliano, J. Warren, and M. McDowell for engineering and technical assistance; M. Furman and S. Heifets for suggesting the mechanism for the general BIM condition; L. Emery and B. Yang for assistance with carrying out electron-cloud-induced beam instability measurements; M. Furman, M. Pivi, and R. Macek for stimulating discussions; L. Loiacono for assistance with BIM calculations; and R. Kustom and J. Noonan for reviewing the manuscript. This work was supported by the U.S. Department of Energy, Office of Basic Energy Sciences under Contract No. W-31-109-ENG-38. 
[1] M. Izawa, Y. Sato, and T. Toyomasu, Phys. Rev. Lett. 74, 5044 (1995).

[2] Z.Y. Guo, K. Ohmi, and H. Fukuma, in Proceedings of the 1998 European Particle Accelerator Conference, Stockholm, Sweden, edited by S. Myers, L. Liljeby, C. Petit-Jean-Genaz, and K.-G. Rensfeld (Institute of Physics, Bristol, UK, 1998), p. 957; Z.Y. Guo, H. Huang, S. P. Li, D. K. Liu, L. Ma, Q. Qin, L. F. Wang, J. Q. Wang, S. H. Wang, C. Zhang, F. Zhou, Y. H. Chin, H. Fukuma, S. Hiramatsu, M. Izawa, T. Kasuga, E. Kikutani, Y. Kobayashi, S. Kurokawa, K. Ohmi, Y. Sato, Y. Suetsugu, M. Tobiyama, K. Yokoya, and X. L. Zhang, in Proceedings of the 1997 Particle Accelerator Conference, Vancouver, edited by M. Comyn, M. K. Craddock, M. Reiser, and J. Thomson (IEEE, Piscataway, NJ, 1998), p. 1566 (http://www. jacow.org).

[3] K. Harkay, R. Rosenberg, Z.Y. Guo, and Q. Qin, in Proceedings of the 2001 Particle Accelerator Conference, Chicago, edited by P. Lucas and S. Webber (IEEE, Piscataway, NJ, 2001), p. 671 (http://www. jacow.org).

[4] R. J. Macek, A. Browman, D. Fitzgerald, R. C. McCrady, F. E. Merrill, M. A. Plum, T. Spickermann, T.-S. Wang, K. C. Harkay, R. Kustom, R. A. Rosenberg, J. E. Griffin, K. Y. Ng, and D. Wildman, in Proceedings of the 2001 Particle Accelerator Conference, Chicago (Ref. [3]), p. 688.

[5] A. Kulikov, A. S. Fisher, S. Heifets, J.T. Seeman, M. Sullivan, U. Wienands, and W. Kozanecki, in Proceedings of the 2001 Particle Accelerator Conference, Chicago (Ref. [3]), p. 1903.

[6] H. Fukuma, in Proceedings of the Mini-Workshop on Electron-Cloud Simulations for Positron and Proton Beams, CERN, Geneva, Switzerland, 2002 (CERN Yellow Report No. CERN-2002-001, 2002) (http://slap.cern.ch/collective/ecloud02/) (unpublished).

[7] G. Arduini, P. Baudrenghien, T. Bohl, P. Collier, K. Cornelis, W. Höfle, T. Linnecar, E. Shaposhnikova, J. Tückmantel, and J. Wenninger, in Proceedings of the 2002 European Particle Accelerator Conference, Paris, France, edited by T. Garvey, J. Le Duff, C. Petit-JeanGenaz, J. Poole, and L. Rivkin (CERN, Geneva, 2002), p. 206; G. Arduini, J. M. Jimenez, and K. Weiss, in Proceedings of the 2001 Particle Accelerator Conference, Chicago (Ref. [3]), p. 685; G. Arduini, V. Baglin, O. Bruning, R. Cappi, F. Caspers, P. Collier, I. R. Collins, K. Cornelis, R. Garoby, O. Grobner, B. Henrist, N. Hilleret, W. Hofle, J. M. Jiminez, J.-M. Laurent, T. Linnecar, E. Mercier, M. Pivi, F. Ruggiero, G. Rumolo, C. Scheuerlein, J. Tuckmantel, L. Voc, and F. Zimmermann, in Proceedings of the 2000 European Particle Accelerator Conference, Vienna, edited by J. L. Laclare, W. Mitaroff, C. Petit-JeanGenaz, J. Poole, and M. Regler (CERN, Geneva, 2000), p. 259; G. Arduini, K. Cornelis, O. Grobner, N. Hilleret, W. Hofle, J. M. Jiminez, J. M. Laurent, G. Moulard, M. Pivi, and K. Weiss, in ibid., p. 939 (http:// www.jacow.org).
[8] M. A. Furman and M. T. F. Pivi, Phys. Rev. ST Accel. Beams 5, 124404 (2002).

[9] K. Ohmi and F. Zimmermann, Phys. Rev. Lett. 85, 3821 (2000); K. Ohmi, Phys. Rev. Lett. 75, 1526 (1995).

[10] M. A. Furman, in Handbook of Accelerator Physics and Engineering, edited by A.W. Chao and M. Tigner (World Scientific, Singapore, 1999), Sec. 2.5.11.

[11] F. Zimmermann, in Proceedings of the 2001 Particle Accelerator Conference, Chicago (Ref. [3]), p. 666; in Proceedings of the 2002 European Particle Accelerator Conference, Paris, France (Ref. [7]), p. 25.

[12] G. Arduini, in Proceedings of the 2002 European Particle Accelerator Conference, Paris, France (Ref. [7]), p. 94.

[13] R. A. Rosenberg and K. C. Harkay, Nucl. Instrum. Methods Phys. Res., Sect. A 453, 507 (2000).

[14] K. C. Harkay and R. A. Rosenberg, in Proceedings of the 1999 IEEE Particle Accelerator Conference, New York, edited by A. Luccio and W. MacKay (IEEE, Piscataway, NJ, 1999), p. 1641 (http://www.jacow.org).

[15] K. C. Harkay, in Proceedings of the 1999 IEEE Particle Accelerator Conference, New York (Ref. [14]), p. 123.

[16] O. Gröbner, in Proceedings of the 10th International Conference on High-Energy Accelerators, Protvino, Russia (Institute of High-Energy Physics, Protvino, 1977), p. 277; in Proceedings of the 1997 Particle Accelerator Conference, Vancouver (Ref. [2]), p. 3589.

[17] V. Baglin, I. Collins, B. Henrist, N. Hilleret, and G. Vorlaufer, CERN Report No. CERN-LHC-ProjectReport-472, 2001 (unpublished).

[18] R. Kirby and F. King, Nucl. Instrum. Methods Phys. Res., Sect. A 469, 1 (2001); also SLAC Report No. SLACPUB-8212, 2000.

[19] R. A. Rosenberg, M.W. McDowell, Q. Ma, and K. C. Harkay (to be published).

[20] J. Galayda (private communication).

[21] R. A. Rosenberg and K. C. Harkay, in Proceedings of the 2001 Particle Accelerator Conference, Chicago (Ref. [3]), p. 2069.

[22] H. Bruining, Physics and Applications of Secondary Electron Emission (McGraw-Hill Book Co., New York, 1954).

[23] H. Seiler, J. Appl. Phys. 54, R1-R18 (1983).

[24] R. Cimino, I. R. Collins, and V. Baglin, Phys. Rev. ST Accel. Beams 2, 063201 (1999).

[25] M. A. Furman, M. Pivi, K. C. Harkay, and R. A. Rosenberg, in Proceedings of the 2001 Particle Accelerator Conference, Chicago (Ref. [3]), p. 679.

[26] P. A. Redhead, J. P. Hobson, and E.V. Kornelsen, The Physical Basis of Ultrahigh Vacuum (Chapman and Hall, London, 1968), Chap. 4 (reprinted by the AIP in 1993 as part of the American Vacuum Society Classics series).

[27] M. Furman and S. Heifets (private communication).

[28] K. C. Harkay, R. A. Rosenberg, M. A. Furman, and M. Pivi, in Proceedings of the Mini-Workshop on Electron-Cloud Simulations for Positron and Proton Beams, CERN, Geneva, Switzerland, 2002 (Ref. [6]). 